\title{
Containing High Order Compact Scheme Source of Steady Convection-Diffusion Equation
}

\author{
Rongfei Wang*, Weihua Wang, Jinhong Yang \\ Institute of Applied Physics, Army Officer Acadamy, Hefei, Anhui \\ Email address: \\ 18655053356@163.com (Rongfei Wang), whwang@ipp.ac.cn (Weihua Wang), yjh_pbxy@sina.com (Jinhong Yang) \\ *Corresponding author
}

\section{To cite this article:}

Rongfei Wang, Weihua Wang, Jinhong Yang. Containing High Order Compact Scheme Source of Steady Convection-Diffusion Equation. Science Discovery. Vol. 4, No. 2, 2016, pp. 156-160. doi: 10.11648/j.sd.20160402.27

Received: February 1, 2016; Accepted: April 28, 2016; Published: June 12, 2016

\begin{abstract}
Convection-diffusion equation have a wide range of applications in many practical engineering problems, such as magnetic confinement fusion problems, heat transfer, particle diffusion. Traditional solutionof convection-diffusion equation in magnetic confinement fusion is Crank-Nicolson scheme. This paper presents a new numerical solution of one-dimensional steady-containing source convection diffusion equation high accuracy difference schemes $\mathrm{O}\left(\tau^{2}+\mathrm{h}^{4}\right)$, which proved to be unconditionally stable using Fourier analysis, numerical experiments show the accuracy and robustness of this format, this scheme has a higher accuracy.
\end{abstract}

Keywords: Convection-Diffusion Equation, Compact Difference, High Order

\section{含源定常对流扩散方程的高精度紧致差分格式}

\author{
王荣飞*, 汪卫华, 杨锦宏 \\ 应用物理研究所, 陆军军官学院, 合肥, 中国
}

邮箱

18655053356@163. com（王荣飞）, whwang@ipp. ac. cn（汪卫华）, y jh_pbxy@sina.com（杨锦宏）

摘要：对流扩散方程在许多工程实际问题中都有着广泛的应用, 如磁约束聚变, 热传导, 粒子扩散等问题。传统的磁 约束聚变的数值模拟中对流扩散方程的差分采用的是Crank-Nicolson格式, 本文提出了一种新的数值求解一维含源定 常对流扩散方程高精度 $\mathrm{O}\left(\tau^{2}+\mathrm{h}^{4}\right)$ 差分格式, 利用Fourier分析法证明该格式是无条件稳定的, 数值实验证明了本文格 式的精确性和稳定性, 该方法具有更高的计算精度。

关键词: 对流扩散方程, 紧致差分, 高精度

\section{1. 引言}

对流扩散方程在许多工程实际问题中 都有着广泛的 应用，如热传导，粒子扩散等问题。有限差分方法常被用 于该问题的数值求解，如中心差分格式，Dufort-Franket
差分格式, 指数型差分格式, Samarski 差分格式, Crank-Nicolson格式, 文章 [1] 将指数变换应用于对流扩 散方程, 把方程转化为线性扩散方程的齐次Neumann边值 问题, 对空间采用四阶紧致差分逼近格式, 时间变量应用 二级四阶Runge-Kutta方法构造出一维对流扩散方程的差 
分格式。文章 [2] 中用时间离散方法对对流扩散方程的求 解得到了空间方向具有七阶精度, 时间方向具有二阶精度 的差分格式。

受以上文献启发, 本文引入指数变换 $\mathrm{u}=\mathrm{m} \cdot \exp [\mathrm{ax} /(2 \varepsilon)], \mathrm{m}=\mathrm{v} \cdot \exp (\mathrm{bt})$ 构造出了一种新的 一维定常对流扩散方程时间二阶、空间四阶精度格式, 然 后证明该格式是无条件稳定的, 并给出了数值算例。

考虑一维非定常含源对流扩散方程:

$$
\left\{\begin{array}{l}
\frac{\partial u}{\partial t}+a \frac{\partial u}{\partial x}=\varepsilon \frac{\partial^{2} u}{\partial x^{2}}+F(x, t) \\
u(x, 0)=d(x), 0<x<1 \\
u(0, t)=g_{0}(x), u(1, t)=g_{1}(x), t>1
\end{array}\right.
$$

其中, $u(x, t)$ 为待求未知量, $v>0$ 为扩散系数, $a \neq 0$ 为对流系数, $\mathrm{f}(\mathrm{x}, \mathrm{t})$ 为非齐次项, 物理上也叫做源汇项, $\mathrm{g}_{0}(\mathrm{x}), \mathrm{g}_{1}(\mathrm{x}), \mathrm{d}(\mathrm{x})$ 均为已知函数。

\section{2. 高精度差分格式的建立与求解}

首先将其区域 $(0,1) \times(0, \mathrm{~T})$ 剖分成差分网格, 记 $\mathrm{h}$ 和 $\tau$ 分别是 $\mathrm{x}$ 方向和 $\mathrm{t}$ 方向的网格步长, 其中 $\mathrm{h}=1 /(\mathrm{N}+1), \mathrm{N}$ 表示内点的个数, $x_{i}=i h, i=0,1, \ldots, N+1$, $\mathrm{t}_{\mathrm{k}}=\mathrm{k} \tau, \mathrm{k}=0,1, \ldots, \quad \mathrm{t}_{\mathrm{k}+1 / 2}=(\mathrm{k}+1 / 2) \tau, \quad \mathrm{u}_{\mathrm{ik}}$ 表示对流扩散 方程 (1) 在离散点 $\left(\mathrm{x}_{\mathrm{i}}, \mathrm{t}_{\mathrm{k}}\right)$ 处的数值解。引入指数变换:

$$
\mathrm{u}=\mathrm{m} \cdot \exp [\mathrm{ax} /(2 \varepsilon)]
$$

将以上变换代入到输运方程（1）中，则原方程可以 化为扩散反应方程:

$$
\frac{\partial \mathrm{m}}{\partial \mathrm{t}}=\mathrm{bm}+\varepsilon \frac{\partial^{2} \mathrm{~m}}{\partial \mathrm{x}^{2}}+\mathrm{s}
$$

其中, $b=-a^{2} /(4 \varepsilon), \quad s=F \cdot \exp [-a x /(2 \varepsilon)]$, 再令

$$
\mathrm{m}=\mathrm{v} \cdot \exp (\mathrm{bt})
$$

将（4）代入（3）, 整理可得:

$$
\frac{\partial \mathrm{v}}{\partial \mathrm{t}}=\varepsilon \frac{\partial^{2} \mathrm{v}}{\partial \mathrm{x}^{2}}+\mathrm{f}
$$

其中, $\mathrm{f}=\mathrm{e}^{-\mathrm{bt}} \mathrm{s}$ 。令:

$$
\mathrm{w}=\frac{\partial^{2} \mathrm{v}}{\partial \mathrm{x}^{2}}
$$

则由（5）式，有

$$
\mathrm{w}=\frac{1}{\varepsilon}\left[\frac{\partial \mathrm{v}}{\partial \mathrm{t}}-\mathrm{f}(\mathrm{x}, \mathrm{t})\right]
$$

定义网格函数: $\mathrm{V}_{\mathrm{i}}^{\mathrm{k}}=\mathrm{v}\left(\mathrm{x}_{\mathrm{i}}, \mathrm{t}_{\mathrm{k}}\right), \mathrm{W}_{\mathrm{i}}^{\mathrm{k}}=\mathrm{w}\left(\mathrm{x}_{\mathrm{i}}, \mathrm{t}_{\mathrm{k}}\right)$, $0 \leq \mathrm{i} \leq \mathrm{m}, \quad 0 \leq \mathrm{k} \leq \mathrm{n}$ 。

由泰勒展开式可得:

$$
\begin{aligned}
& \delta_{\mathrm{x}}{ }^{2} \mathrm{~V}_{\mathrm{i}}^{\mathrm{k}}=\frac{\mathrm{v}_{\mathrm{i}+1}{ }^{\mathrm{k}}-2 \mathrm{v}_{\mathrm{i}}{ }^{\mathrm{k}}+\mathrm{v}_{\mathrm{i}-1}{ }^{\mathrm{k}}}{\mathrm{h}^{2}}=\frac{\partial^{2} \mathrm{v}}{\partial \mathrm{x}^{2}}\left(\mathrm{x}_{\mathrm{i}}, \mathrm{t}_{\mathrm{k}}\right)+\frac{\mathrm{h}^{2}}{12} \frac{\partial^{4} \mathrm{v}}{\partial \mathrm{x}^{4}}\left(\mathrm{x}_{\mathrm{i}}, \mathrm{t}_{\mathrm{k}}\right)+\frac{\mathrm{h}^{4}}{360} \frac{\partial^{6} \mathrm{v}}{\partial \mathrm{x}^{6}}\left(\xi_{\mathrm{ik}}, \mathrm{t}_{\mathrm{k}}\right)=\mathrm{v}\left(\mathrm{x}_{\mathrm{i}}, \mathrm{t}_{\mathrm{k}}\right)+\frac{\mathrm{h}^{2}}{12} \frac{\partial^{2} \mathrm{w}}{\partial \mathrm{x}^{2}}\left(\mathrm{x}_{\mathrm{i}}, \mathrm{t}_{\mathrm{k}}\right)+\frac{\mathrm{h}^{4}}{360} \frac{\partial^{6} \mathrm{v}}{\partial \mathrm{x}^{6}}\left(\xi_{\mathrm{ik}}, \mathrm{t}_{\mathrm{k}}\right) \\
= & \mathrm{w}_{\mathrm{i}}{ }^{\mathrm{k}}+\frac{\mathrm{h}^{2}}{12}\left[\delta_{\mathrm{x}}{ }^{2} \mathrm{~W}_{\mathrm{i}}^{\mathrm{k}}-\frac{\mathrm{h}^{2}}{12} \frac{\partial^{4} \mathrm{w}}{\partial \mathrm{x}^{4}}\left(\eta_{\mathrm{ik}}, \mathrm{t}_{\mathrm{k}}\right)\right]+\frac{\mathrm{h}^{4}}{360} \frac{\partial^{6} \mathrm{v}}{\partial \mathrm{x}^{6}}\left(\xi_{\mathrm{ik}}, \mathrm{t}_{\mathrm{k}}\right)=\frac{1}{12}\left(\mathrm{~W}_{\mathrm{i}-1}{ }^{\mathrm{k}}+\mathrm{W}_{\mathrm{i}}^{\mathrm{k}}+\mathrm{W}_{\mathrm{i}+1}{ }^{\mathrm{k}}\right)+\left[\frac{1}{360} \frac{\partial^{6} \mathrm{v}}{\partial \mathrm{x}^{6}}\left(\xi_{\mathrm{ik}}, \mathrm{t}_{\mathrm{k}}\right)-\frac{\mathrm{h}^{2}}{144} \frac{\partial^{6} \mathrm{v}}{\partial \mathrm{x}^{6}}\left(\eta_{\mathrm{ik}}, \mathrm{t}_{\mathrm{k}}\right)\right] \mathrm{h}^{4},
\end{aligned}
$$

其中 $\xi_{\mathrm{ik}}, \eta_{\mathrm{ik}} \in\left(\mathrm{x}_{\mathrm{i}-1}, \mathrm{x}_{\mathrm{i}+1}\right)$ 。将上式中上标为 $\mathrm{k}$ 和 $\mathrm{k}+1$ 的两个等式相加并除以 2 , 应用公式 $\mathrm{W}_{\mathrm{i}}^{\mathrm{k}+\frac{1}{2}}=\frac{\mathrm{w}\left(\mathrm{x}_{\mathrm{i}}, \mathrm{t}_{\mathrm{k}}\right)+\mathrm{w}\left(\mathrm{x}_{\mathrm{i}}, \mathrm{t}_{\mathrm{k}+1}\right)}{2}=\mathrm{w}\left(\mathrm{x}_{\mathrm{i}}, \mathrm{t}_{\mathrm{k}+1 / 2}\right)+\frac{\tau^{2}}{8} \frac{\partial^{2} \mathrm{w}}{\partial \mathrm{t}^{2}}\left(\mathrm{x}_{\mathrm{i}}, \theta_{\mathrm{ik}}\right)$, 可得:

$$
\begin{aligned}
\frac{1}{2}\left(\delta_{\mathrm{x}}{ }^{2} \mathrm{~V}_{\mathrm{i}}^{\mathrm{k}}+\delta_{\mathrm{x}}{ }^{2} \mathrm{~V}_{\mathrm{i}}^{\mathrm{k}+1}\right)=\frac{1}{12}\left(\mathrm{~W}_{\mathrm{i}-1}{ }^{\mathrm{k}+\frac{1}{2}}+\mathrm{W}_{\mathrm{i}}^{\mathrm{k}+\frac{1}{2}}+\mathrm{W}_{\mathrm{i}+1}{ }^{\mathrm{k}+\frac{1}{2}}\right)+\left[\frac{1}{360} \frac{\partial^{6} \mathrm{v}}{\partial \mathrm{x}^{6}}\left(\bar{\xi}_{\mathrm{ik}}, \overline{\mathrm{t}}_{\mathrm{k}}\right)-\frac{\mathrm{h}^{2}}{144} \frac{\partial^{6} \mathrm{v}}{\partial \mathrm{x}^{6}}\left(\bar{\eta}_{\mathrm{ik}}, \overline{\mathrm{t}}_{\mathrm{k}}\right)\right] \mathrm{h}^{4} \\
=\frac{1}{12}\left[\mathrm{w}\left(\mathrm{x}_{\mathrm{i}-1}, \mathrm{t}_{\mathrm{k}+1 / 2}\right)+10 \mathrm{w}\left(\mathrm{x}_{\mathrm{i}}, \mathrm{t}_{\mathrm{k}+1 / 2}\right)+\mathrm{w}\left(\mathrm{x}_{\mathrm{i}+1}, \mathrm{t}_{\mathrm{k}+1 / 2}\right)\right]+\frac{\tau^{2}}{8} \cdot \frac{1}{12} \cdot\left[\frac{\partial^{2} \mathrm{w}}{\partial \mathrm{t}^{2}}\left(\mathrm{x}_{\mathrm{i}-1}, \theta_{\mathrm{ik}}\right)+10 \frac{\partial^{2} \mathrm{w}}{\partial \mathrm{t}^{2}}\left(\mathrm{x}_{\mathrm{i}}, \theta_{\mathrm{ik}}\right)+\frac{\partial^{2} \mathrm{w}}{\partial \mathrm{t}^{2}}\left(\mathrm{x}_{\mathrm{i}+1}, \theta_{\mathrm{ik}}\right)\right] \\
+\left[\frac{1}{360} \frac{\partial^{6} \mathrm{v}}{\partial \mathrm{x}^{6}}\left(\bar{\xi}_{\mathrm{ik}}, \overline{\mathrm{t}}_{\mathrm{k}}\right)-\frac{\mathrm{h}^{2}}{144} \frac{\partial^{6} \mathrm{v}}{\partial \mathrm{x}^{6}}\left(\bar{\eta}_{\mathrm{ik}}, \overline{\mathrm{t}}_{\mathrm{k}}\right)\right] \mathrm{h}^{4}
\end{aligned}
$$

利用（7），应用公式 $\frac{\partial v}{\partial t}\left(x_{i}, t_{k+1 / 2}\right)=\delta_{t} V_{i}^{k+1 / 2}-\frac{\tau^{2}}{24} \cdot \frac{\partial^{3} v}{\partial t^{3}}\left(x_{i}, \vartheta_{i k}\right)$ 得到

$$
\delta_{x}^{2} V_{i}^{k+1 / 2}=\frac{1}{\varepsilon} \cdot \frac{1}{12}\left(\delta_{t} V_{i-1}^{k+1 / 2}+10 \delta_{t} V_{i}^{k+1 / 2}+\delta_{t} V_{i+1}^{k+1 / 2}\right)
$$




$$
\begin{gathered}
-\frac{1}{\varepsilon} \cdot \frac{1}{12}\left(\mathrm{f}\left(\mathrm{x}_{\mathrm{i}-1}, \mathrm{t}_{\mathrm{k}+1 / 2}\right)+10 \mathrm{f}\left(\mathrm{x}_{\mathrm{i}}, \mathrm{t}_{\mathrm{k}+1 / 2}\right)+\mathrm{f}\left(\mathrm{x}_{\mathrm{i}+1}, \mathrm{t}_{\mathrm{k}+1 / 2}\right)\right)-\frac{1}{\varepsilon} \cdot \frac{\tau^{2}}{24} \cdot \frac{1}{12} \cdot\left[\frac{\partial^{3} \mathrm{v}}{\partial \mathrm{t}^{3}}\left(\mathrm{x}_{\mathrm{i}-1}, \vartheta_{\mathrm{ik}}\right)+10 \frac{\partial^{3} \mathrm{v}}{\partial \mathrm{t}^{3}}\left(\mathrm{x}_{\mathrm{i}}, \vartheta_{\mathrm{ik}}\right)+\frac{\partial^{3} \mathrm{v}}{\partial \mathrm{t}^{3}}\left(\mathrm{x}_{\mathrm{i}+1}, \vartheta_{\mathrm{ik}}\right)\right] \\
+\frac{\tau^{2}}{8} \cdot \frac{1}{12} \cdot\left[\frac{\partial^{4} \mathrm{v}}{\partial \mathrm{x}^{2} \partial \mathrm{t}^{2}}\left(\mathrm{x}_{\mathrm{i}-1}, \theta_{\mathrm{ik}}\right)+10 \frac{\partial^{4} \mathrm{v}}{\partial \mathrm{x}^{2} \partial \mathrm{t}^{2}}\left(\mathrm{x}_{\mathrm{i}}, \theta_{\mathrm{ik}}\right)+\frac{\partial^{4} \mathrm{v}}{\partial \mathrm{x}^{2} \partial \mathrm{t}^{2}}\left(\mathrm{x}_{\mathrm{i}+1}, \theta_{\mathrm{ik}}\right)\right]+\left[\frac{1}{360} \frac{\partial^{6} \mathrm{v}}{\partial \mathrm{x}^{6}}\left(\bar{\xi}_{\mathrm{ik}}, \overline{\mathrm{t}}_{\mathrm{k}}\right)-\frac{\mathrm{h}^{2}}{144} \frac{\partial^{6} \mathrm{v}}{\partial \mathrm{x}^{6}}\left(\bar{\eta}_{\mathrm{ik}}, \overline{\mathrm{t}}_{\mathrm{k}}\right)\right] \mathrm{h}^{4}, \\
\begin{array}{c}
\text { 其中 } \\
\text { 记 }
\end{array} \overline{\mathrm{t}}_{\mathrm{k}} \in\left(\mathrm{x}_{\mathrm{i}-1}, \mathrm{x}_{\mathrm{i}+1}\right), \overline{\mathrm{t}}_{\mathrm{k}}, \theta_{\mathrm{ik}}, \vartheta_{\mathrm{ik}} \in\left(\mathrm{t}_{\mathrm{k}}, \mathrm{t}_{\mathrm{k}+1}\right) 。 \\
\mathrm{R}_{\mathrm{ik}}=\varepsilon\left[\frac{\mathrm{h}^{2}}{144} \frac{\partial^{6} \mathrm{v}}{\partial \mathrm{x}^{6}}\left(\bar{\eta}_{\mathrm{ik}}, \overline{\mathrm{t}}_{\mathrm{k}}\right)-\frac{1}{360} \frac{\partial^{6} \mathrm{v}}{\partial \mathrm{x}^{6}}\left(\bar{\xi}_{\mathrm{ik}}, \overline{\mathrm{t}}_{\mathrm{k}}\right)\right] \mathrm{h}^{4}+\frac{\tau^{2}}{24} \cdot \frac{1}{12} \cdot\left[\frac{\partial^{3} \mathrm{v}}{\partial \mathrm{t}^{3}}\left(\mathrm{x}_{\mathrm{i}-1}, \vartheta_{\mathrm{ik}}\right)+10 \frac{\partial^{3} \mathrm{v}}{\partial \mathrm{t}^{3}}\left(\mathrm{x}_{\mathrm{i}}, \vartheta_{\mathrm{ik}}\right)+\frac{\partial^{3} \mathrm{v}}{\partial \mathrm{t}^{3}}\left(\mathrm{x}_{\mathrm{i}+1}, \vartheta_{\mathrm{ik}}\right)\right] \\
+\frac{\tau^{2}}{8} \cdot \frac{\varepsilon}{12} \cdot\left[\frac{\partial^{4} \mathrm{v}}{\partial \mathrm{x}^{2} \partial \mathrm{t}^{2}}\left(\mathrm{x}_{\mathrm{i}-1}, \theta_{\mathrm{ik}}\right)+10 \frac{\partial^{4} \mathrm{v}}{\partial \mathrm{x}^{2} \partial \mathrm{t}^{2}}\left(\mathrm{x}_{\mathrm{i}}, \theta_{\mathrm{ik}}\right)+\frac{\partial^{4} \mathrm{v}}{\partial \mathrm{x}^{2} \partial \mathrm{t}^{2}}\left(\mathrm{x}_{\mathrm{i}+1}, \theta_{\mathrm{ik}}\right)\right]
\end{gathered}
$$

略去小量项 $R_{i k}$, 并且用 $v_{i, k}$ 表示 $V_{i}{ }^{k}$ 的近似值, 得到下列差分格式

$$
\begin{gathered}
\frac{1}{12}\left(\delta_{\mathrm{t}} \mathrm{v}_{\mathrm{i}-1}{ }^{\mathrm{k}+1 / 2}+10 \delta_{\mathrm{t}} \mathrm{v}_{\mathrm{i}}^{\mathrm{k}+1 / 2}+\delta_{\mathrm{t}} \mathrm{v}_{\mathrm{i}+1}{ }^{\mathrm{k}+1 / 2}\right)-\varepsilon \delta_{\mathrm{x}}{ }^{2} \mathrm{v}_{\mathrm{i}}^{\mathrm{k}+1 / 2} \\
=\frac{1}{12}\left[\mathrm{f}\left(\mathrm{x}_{\mathrm{i}-1}, \mathrm{t}_{\mathrm{k}+1 / 2}\right)+10 \mathrm{f}\left(\mathrm{x}_{\mathrm{i}}, \mathrm{t}_{\mathrm{k}+1 / 2}\right)+\mathrm{f}\left(\mathrm{x}_{\mathrm{i}+1}, \mathrm{t}_{\mathrm{k}+1 / 2}\right)\right] \\
1 \leq \mathrm{i} \leq \mathrm{m}-1, \quad 0 \leq \mathrm{k} \leq \mathrm{n}-1 . \\
\mathrm{V}_{\mathrm{i}}{ }^{0}=\varphi\left(\mathrm{x}_{\mathrm{i}}\right), \quad 1 \leq \mathrm{i} \leq \mathrm{m}, \\
\mathrm{V}_{0}{ }^{\mathrm{k}}=\partial\left(\mathrm{t}_{\mathrm{k}}\right), \quad \mathrm{V}_{\mathrm{m}}{ }^{\mathrm{k}}=\beta\left(\mathrm{t}_{\mathrm{k}}\right), \quad 1 \leq \mathrm{k} \leq \mathrm{m},
\end{gathered}
$$

记

$$
\mathrm{c}=\max \left\{\frac{1}{24} \max _{\substack{0 \leq x \leq 1 \\ 0 \leq \mathrm{t} \leq \mathrm{T}}}\left|\frac{\partial^{3} \mathrm{v}(\mathrm{x}, \mathrm{t})}{\partial \mathrm{t}^{3}}\right|+\frac{\varepsilon}{8} \max _{\substack{0 \leq x \leq 1 \\ 0 \leq \mathrm{T} \leq \mathrm{T}}}\left|\frac{\partial^{4} \mathrm{v}(\mathrm{x}, \mathrm{t})}{\partial \mathrm{x}^{2} \partial \mathrm{t}^{2}}\right|, \frac{7 \varepsilon}{720} \max _{\substack{0 \leq \mathrm{x} \leq 1 \\ 0 \leq \mathrm{T} \leq \mathrm{T}}}\left|\frac{\partial^{6} \mathrm{v}(\mathrm{x}, \mathrm{t})}{\partial \mathrm{t}^{6}}\right|\right\},
$$

则差分格式 (10) 式的局部截断误差 $\mathrm{R}_{\mathrm{ik}}$, 满足

$$
a_{i-1} u_{i-1, k+1}+a_{i} u_{i, k+1}+a_{i+1} u_{i+1, k+1}=b_{i-1} u_{i-1, k}+b_{i} u_{i, k+1}+b_{i+1} u_{i+1, k}+c_{i}
$$

$\mathrm{R}_{\mathrm{ik}} \leq \mathrm{c}\left(\tau^{2}+\mathrm{h}^{4}\right), 1 \leq \mathrm{i} \leq \mathrm{m}-1,0 \leq \mathrm{k} \leq \mathrm{n}-1$.

差分格式 (10) 的结点图如图1所示, 它也是一个2 层格式。

\section{其中}

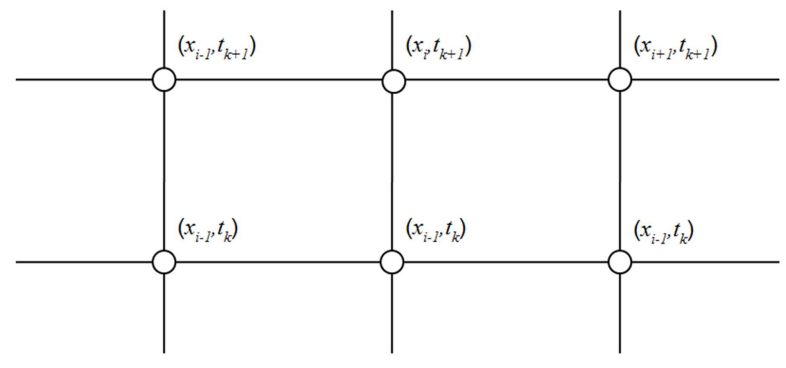

图 1 紧致差分的结点图。

$$
\begin{gathered}
a_{i-1}=(1-6 \varepsilon r) \exp \left\{\frac{a^{2} \tau}{4 \varepsilon}+\frac{a h}{2 \varepsilon}\right\}, \\
a_{i}=2(5+6 \varepsilon r) \exp \left\{\frac{a^{2} \tau}{4 \varepsilon}\right\}, \\
a_{i+1}=(1-6 \varepsilon r) \exp \left\{\frac{a^{2} \tau}{4 \varepsilon}-\frac{a h}{2 \varepsilon}\right\}, \\
b_{i-1}=(1+6 \varepsilon r) \exp \left\{\frac{a h}{2 \varepsilon}\right\}, \\
b_{i}=2(5-6 \varepsilon r),
\end{gathered}
$$

且令 $r=\tau / h^{2}$, 可推导出来方程 (1) 的四阶精度隐式差 分格式 


$$
\begin{gathered}
\mathrm{b}_{\mathrm{i}+1}=(1+6 \mathrm{vr}) \exp \left\{-\frac{\alpha \mathrm{h}}{2 \mathrm{v}}\right\}, \\
\mathrm{c}_{\mathrm{i}}=\frac{\tau}{2} \exp \left\{\frac{\mathrm{a}^{2} \tau}{4 \varepsilon}+\frac{\mathrm{ah}}{2 \varepsilon}\right\} \mathrm{f}_{\mathrm{i}-1, \mathrm{k}+1}+5 \tau \exp \left\{\frac{\mathrm{a}^{2} \tau}{4 \varepsilon}\right\} \mathrm{f}_{\mathrm{i}, \mathrm{k}+1} \\
+\frac{\tau}{2} \exp \left\{\frac{\mathrm{a}^{2} \tau}{4 \varepsilon}-\frac{a h}{2 \varepsilon}\right\} \mathrm{f}_{\mathrm{i}+1, \mathrm{k}+1}+\frac{\tau}{2} \exp \left\{\frac{\mathrm{ah}}{2 \varepsilon}\right\} \mathrm{f}_{\mathrm{i}-1, \mathrm{k}} \\
+5 \tau \mathrm{f}_{\mathrm{i}, \mathrm{k}}+\exp \left\{-\frac{\mathrm{ah}}{2 \varepsilon}\right\} \mathrm{f}_{\mathrm{i}+1, \mathrm{k}}
\end{gathered}
$$

由差分格式的推导过程可以知道, 该两层三点差分格 式的截断误差为 $\mathrm{O}\left(\tau^{2}+\mathrm{h}^{4}\right)$ 。

在格式 (13) 中, 令空间下标为 $i=1,2, \ldots, N$, 便可以 得到差分方程组的矩阵形式 $\mathrm{Au}_{\mathrm{k}+1}=\mathrm{Bu}_{\mathrm{k}}+\mathrm{RH}$

其中

$$
\begin{aligned}
\mathrm{A} & =\left[\begin{array}{cccccc}
\mathrm{a} & \mathrm{b} & & & & \\
\mathrm{c} & \mathrm{a} & \mathrm{b} & & & \\
& & \ldots & \ldots & \ldots & \\
& & & \mathrm{c} & \mathrm{a} & \mathrm{b} \\
& & & & \mathrm{c} & \mathrm{a}
\end{array}\right]_{\mathrm{M} \times \mathrm{N}} \\
\mathrm{B} & =\left[\begin{array}{cccccc}
\overline{\mathrm{a}} & \overline{\mathrm{b}} & & & & \\
\overline{\mathrm{c}} & \overline{\mathrm{a}} & \overline{\mathrm{b}} & & & \\
& & \ldots & \ldots & \ldots & \\
& & & \bar{c} & \overline{\mathrm{a}} & \overline{\mathrm{b}} \\
& & & & \overline{\mathrm{c}} & \overline{\mathrm{a}}
\end{array}\right]_{\mathrm{M} \times \mathrm{N}}
\end{aligned}
$$

$$
\left\{\begin{array}{l}
\mathrm{a}=2(5+6 \varepsilon \mathrm{r}) \exp \left[\mathrm{a}^{2} \tau /(4 \varepsilon)\right] \\
\mathrm{b}=(1-6 \varepsilon \mathrm{r}) \exp \left[\mathrm{a}^{2} \tau /(4 \varepsilon)-\mathrm{ah} /(2 \varepsilon)\right] \\
\mathrm{c}=(1-6 \varepsilon \mathrm{r}) \exp \left[\mathrm{a}^{2} \tau /(4 \varepsilon)+\mathrm{ah} /(2 \varepsilon)\right] \\
\overline{\mathrm{a}}=2(5-6 \varepsilon \mathrm{r}) \\
\overline{\mathrm{b}}=(1+6 \varepsilon \mathrm{r}) \exp [-\mathrm{ah} /(2 \varepsilon)] \\
\mathrm{c}=(1+6 \varepsilon \mathrm{r}) \exp [\mathrm{ah} /(2 \varepsilon)]
\end{array}\right.
$$

$\mathrm{k} \tau$ 时刻的解向量为 $\mathrm{u}_{\mathrm{k}}$, 表示为

$$
\mathrm{u}_{\mathrm{k}}=\left[\begin{array}{c}
\mathrm{u}_{1, \mathrm{k}} \\
\mathrm{u}_{2, \mathrm{k}} \\
\vdots \\
\mathrm{u}_{\mathrm{N}, \mathrm{k}}
\end{array}\right]
$$

\section{3. 稳定性分析}

假设边界条件精确满足, 并且右端项 $\mathrm{f}$ 无误差存在, 采用离散的Fourier分析方法对格式 (10) 进行稳定性分析:

$$
\begin{aligned}
& A_{j-1} u_{j-1}{ }^{n+1}+A_{j} u_{j}{ }^{n+1}+A_{j+1} u_{j+1}{ }^{n+1} \\
& =B_{j-1} u_{j-1}{ }^{n}+B_{j} u_{j}{ }^{n}+B_{j+1} u_{j+1}{ }^{n}
\end{aligned}
$$

用 $v_{j}{ }^{n}$ 表示计算 $u_{j}{ }^{n}$ 所产生的误差, 则 $v_{j}{ }^{n}$ 满足齐次方 程

$$
\begin{aligned}
& A_{j-1} v_{j-1}{ }^{n+1}+A_{j} v_{j}{ }^{n+1}+A_{j+1} v_{j+1}{ }^{n+1} \\
& =B_{j-1} v_{j-1}{ }^{n}+B_{j} v_{j}{ }^{n}+B_{j+1} v_{j+1}{ }^{n}
\end{aligned}
$$

其中:

$$
A_{j-1}=1-6 r, A_{j}=10+12 r, A_{j+1}=1-6 r, B_{j-1}=1+6 r, B_{j}=10-12 r, B_{j+1}=1+6 r
$$

令 $v_{j}{ }^{n}=\lambda^{n} e^{i \sigma j h}$, 其中, $i$ 为虚数单位, $\sigma$ 为波数, 运用关系式 $e^{i x}=\cos x+i \sin x$, 代入（17），得到增长因 子为

\section{4. 数值算例}

$$
G=\frac{\lambda^{n+1}}{\lambda^{n}}=\frac{2 \cos \sigma h+10-12(1-\cos \sigma h)}{2 \cos \sigma h+10+12(1-\cos \sigma h)}
$$

因为 $1-\cos \sigma h \geq 0$,

所以

$$
|\mathrm{G}| \leq 1
$$

即格式 (10) 为无条件稳定的, 同理, (13) 也为无条件 稳定的。

考虑模型:

$$
\left\{\begin{array}{l}
\frac{\partial u}{\partial t}+\frac{\partial u}{\partial x}=v \frac{\partial^{2} u}{\partial x^{2}}+\left(x^{2}-3 x+1+2 v\right) \exp (-t) \\
0<x<1, t>0 \\
u(x, 0)=x(1-x) \\
u(0, t)=u(1, t)=0
\end{array}\right.
$$

其解析解为 $\mathrm{u}(\mathrm{x}, 0)=\mathrm{x}(1-\mathrm{x}) \exp (-\mathrm{t})$ 。表 1 给出了 $h=0.1, \tau=0.01, x=0.5$ 时, 不同差分格式的计算误差随时 间的变化。表 2 给出了取不同步长比时, 紧致差分格式数 值解的最大误差 $E_{\infty}(h, \tau)=\max _{\substack{1 \leq i \leq m-1 \\ 1 \leq k \leq n}}\left|u\left(x_{i}, t_{k}\right)-u_{i}^{k}\right| 。$ 
表1 不同差分格式数值解与精确解的误差变化。

\begin{tabular}{|c|c|c|c|}
\hline 时间 $t$ & (s) & 迎风差分格式 & 中心显式差分格式 \\
\hline 0.1 & & $3.569713 \times 10^{-3}$ & $4.964423 \times 10^{-4}$ \\
\hline 0.2 & & 4. $563294 \times 10^{-3}$ & 7. $963428 \times 10^{-4}$ \\
\hline 0.3 & & $6.913451 \times 10^{-3}$ & $9.964824 \times 10^{-4}$ \\
\hline 0.4 & & $5.621643 \times 10^{-3}$ & 1. $659135 \times 10^{-3}$ \\
\hline 0.5 & & 9. $121533 \times 10^{-3}$ & 1. $033639 \times 10^{-3}$ \\
\hline 0.6 & & $1.003694 \times 10^{-2}$ & 7. $694563 \times 10^{-4}$ \\
\hline 0.7 & & 8. $364695 \times 10^{-3}$ & 7. $546397 \times 10^{-4}$ \\
\hline 0.8 & & 9. $643482 \times 10^{-3}$ & 5. $697213 \times 10^{-4}$ \\
\hline 0.9 & & $6.316181 \times 10^{-3}$ & 3. $200156 \times 10^{-4}$ \\
\hline 1 & & 7. $236458 \times 10^{-3}$ & $7.984543 \times 10^{-4}$ \\
\hline $\mathrm{h}$ & $\tau$ & $\mathrm{E}_{\infty}(\mathrm{h}, \tau)$ & $\mathrm{E}_{\infty}(2 \mathrm{~h}, 2 \tau) / \mathrm{E}_{\infty}(\mathrm{h}, \tau)$ \\
\hline $1 / 10$ & $1 / 100$ & $5.032641 \times 10^{-6}$ & * \\
\hline $1 / 20$ & $1 / 400$ & 3. $072430 \times 10^{-7}$ & 16. 38 \\
\hline $1 / 40$ & $1 / 1600$ & 1. $865471 \times 10^{-8}$ & 16. 47 \\
\hline $1 / 80$ & $1 / 48000$ & 1. $181426 \times 10^{-8}$ & 15. 79 \\
\hline $1 / 160$ & $1 / 19200$ & 7. $288254 \times 10^{-11}$ & 16. 21 \\
\hline
\end{tabular}

经过比较可以看出, 相同条件下本文差分格式的精度 要明显优于其他几种常见的差分格式, 在空间步长缩小到 原来的 $1 / 2$, 时间步长缩小到原来的 $1 / 4$ 时, 最大误差缩小 到原来的 $1 / 16$. 这充分体现了四阶格式的精度特性, 所以 本文的四阶紧致差分格式实现了在较粗网格下获得较高 精度数值解的目的。

\section{5. 结论}

采用指数变换 $\mathrm{u}=\mathrm{m} \cdot \exp [\mathrm{ax} /(2 \varepsilon)]$ 和 $\mathrm{m}=\mathrm{v} \cdot \exp (\mathrm{bt})$, 将对流扩散方程转化为抛物线方程, 对抛物线方程采用紧 致差分格式差分, 使其截断误差为 $\mathrm{O}\left(\tau^{2}+\mathrm{h}^{4}\right)$, 经过证明 该格式是无条件稳定的, 数值算例验证了格式的有效性。 因此, 该差分格式可用于求解磁约束聚变中的对流扩散方 程。

\section{致谢}

本文为基金项目: 国家磁约束核聚变能发展研究专项 项目 (2013GB113004，2015GB121007), 国家自然科学基金 项目（51576208，91326101）的阶段性成果之一。

\begin{tabular}{ll} 
Crank-Nicolson格式 & 四阶紧致差分格式 \\
\hline $6.627795 \times 10^{-5}$ & $1.467825 \times 10^{-6}$ \\
$9.456913 \times 10^{-5}$ & $1.763545 \times 10^{-6}$ \\
$1.635425 \times 10^{-4}$ & $2.635791 \times 10^{-6}$ \\
$1.001472 \times 10^{-4}$ & $3.937543 \times 10^{-6}$ \\
$8.752287 \times 10^{-5}$ & $4.786354 \times 10^{-6}$ \\
$4.375623 \times 10^{-5}$ & $3.556357 \times 10^{-6}$ \\
$3.336874 \times 10^{-5}$ & $2.944531 \times 10^{-6}$ \\
$8.634985 \times 10^{-5}$ & $2.012211 \times 10^{-6}$ \\
$1.675647 \times 10^{-4}$ & $2.867214 \times 10^{-6}$ \\
$9.345633 \times 10^{-5}$ & $3.645697 \times 10^{-6}$ \\
\hline
\end{tabular}

\section{参考文献}

[1] 王慧蓉. 求解对流扩散方程的紧致二级四阶Runge-Kutta差 分格式 $[\mathrm{J}]$. 云南民族大学学报: 自然科学版, 2015, 24 (5) :382-385。

[2] 杨志峰. 含源汇定常对流扩散问题紧致四阶差分格式 [J]. 科学通报, 1991，38(2):113-116。

[3] 孙志忠. 偏微分方程数值解法 [M]. 北京: 科学出版社, 2005。

[4] 钱凌志, 顾海波. 高阶紧致差分格式结合外推技巧求解对流 扩散方程 [J]. 山东大学学报: 理学版, 2011, 46 (12) : 39-43。

[5] 张阳. 线性对流占优扩散问题的交替方向差分流线扩散法 [J]. 计算数学, 2007, 29(1):49-66。

[6] 何文平. 求解对流扩散方程的四中差分格式的比较 [J]. 物 理学报, 2004.(10):3258-3264。

[7］王倩倩, 李金金, 孙启航.一维变系数对流扩散方程的一个紧 致差分格式. 江苏师范大学学报: 自然科学版, 2013, 31 (2) :21-24。

[8］王彩华.一维对流扩散方程的一类新型高精度紧致差分格 式 $[J]$. 水动力学研究与进展: A 辑, 2004, (19):655。

[9] 戴嘉尊. 微分方程数值解法 [M]. 2版. 南京: 东南大学出版社, 2004 。

[10] Patanker S V. Numerical heat transfer and fluid flow [M]. New York: Hemisphere Publishing Corporation, 1980. 